Revista de Psicología de la PUCP. Vol. XX, 1, 2002

\title{
Depresión en personas que viven con VIH
}

\author{
Ninoshka Fasce Cayo \\ Lima
}

El estudio investiga la depresión en un grupo 55 adultos (14 mujeres y 41 hombres) que viven con VIH, entre 18 y 58 años, de nivel socioeconómico bajo y medio bajo que acuden a centros de salud estatales, ONG y grupos de ayuda mutua (GAM) de Lima. Se uso el Inventario de Depresión de Beck para estudiar el nivel de depresión, así como los cinco factores encontrados en esta población. Se correlacionaron los resultados descriptivos con las siguientes variables demográficas: edad, sexo, tiempo de diagnóstico, presencia o no de síntomas asociados al VIH, participación o no en un GAM, orientación sexual y sexo. Los resultados indicaron que la depresión varía según la persona pertenezca o no a un GAM, el sexo y la orientación sexual.

Palabras clave: depresión, Virus de Inmunodeficiencia Humano (VIH), grupo de ayuda mutua (GAM), homosexualidad, heterosexualidad.

\section{Depression in people, that live with HIV in Lima}

The study investigates the depression in a group 55 adults ( 14 women and 41 men) who live with VIH, between 18 and 58 years, of low and medium low socioeconomic level which attend public health centers, NGO and mutual support groups (MSG) of Lima. The Beck Depression Inventory was used to study the level of depression, as well as the five factors found in this population. The descriptive results were correlated with the following demographic variables: age, sex, time of diagnosis, the presence or absence of symptoms associated to VIH, the participation or not in a MSG, sexual orientation and sex. Results indicated that depression varies depending on whether the person belongs or not to a MSG, sex and sexual orientation.

Key words: depression, Virus of Inmunodeficiencia Humano (VIH), mutual support groups (MSG), homosexuality, hetrosexuality.

1 Licenciada en psicología de la PUCP. Estudios de Maestría e la Universidad Peruana Cayetano Heredia. Presidenta de la Asociación UNESCO Warmikuna Perú. Presta asesoría psicológica al Grupo de Ayuda Mutua (Vida y Amor). Se desempeña como gerenta de la Asociación Psicológica Motiva. Su área de interés en investigación se orienta a los factores que afectan la calidad de vida de las personas que viven con VIH. Correo electrónico: ninoshka@terra.com.pe 

El VIH cuenta actualmente con 33.4 millones de casos en el ámbito mundial (OMS, 1998), 1088053 casos en Latinoamérica (OPS, 2001) 22465 en el Perú (Ministerio de Salud, 2001a), ubicándose el $66.9 \%$ en Lima. Estas cifras muestran una tendencia a aumentar geométricamente. Consideramos importante tomar en cuenta la calidad de vida de las personas que viven con el VIH, pues ésta se ve afectada directamente por los efectos del virus sobre el sistema inmune, así como, por sentimientos de culpa, vergüenza y miedo frente a la muerte, lo que trae como consecuencia la depresión (Fasce, 1999; Hays, Turner y Coates, 1992).

Se sabe, gracias a los avances de la psiconeuroinmunología que las emociones negativas, como la depresión, afectan directa y negativamente la respuesta inmunológica, lo que en el caso de los seropositivos es especialmente perjudicial (Fasce; Hays, Turner y Coates). Por ello se considera importante evaluar y describir los niveles de depresión en las personas que viven con VIH para contar con una herramienta con la cual diseñar programas de intervención en los diferentes niveles de la prevención y específica para las diferentes poblaciones.

La mayoría de investigaciones realizadas con la población seropositiva se basan en hombres que tienen sexo con otros hombre (HSH) a pesar de incrementarse cada vez más el numero de heterosexuales seropositivos, especialmente mujeres que en el Perú alcanzan una proporción hombre a mujer de 3 a 1 (Ministerio de Salud, 2001a). Estos datos confirman observaciones respecto a la progresiva feminización de la epidemia por lo que se las considera un grupo vulnerable (Kornblit, 1997, CONTRASIDA, 1998b). Para aquellas que tienen prole, la maternidad y cuidado futuro de los hijos generan preocupación con respecto al futuro, lo que acrecienta las emociones disfóricas como la depresión y la desesperanza. 
Ragúz (1995) plantea que la femineidad, predice falta de asertividad, neuroticismo, baja autoestima, inseguridad, pasividad, temor, dependencia, auto-desprecio, incapacidad aprendida y depresión. Una persona insegura, dependiente, que se percibe ineficaz tenderá más a buscar ayuda, a pedir consejo, a ampararse en su grupo de soporte para resolver los problemas y esperar que alguien con más capacidad y mayor asertividad los resuelvan. Alguien con estas características espera mucho de su red de soporte, las expectativas que tiene de su entorno son muy altas, y por lo tanto difícilmente serán satisfechas, lo que elevaría las probabilidades de deprimirse.

En este sentido, se ha planteado que las mujeres experimentan fortaleza para afrontar sus dificultades mientras tienen hijos menores de cuatro años (Torres, 1998), dado que la motivación de nutrir y cuidar a los demás es mayor que cuando los hijos asisten a la escuela y son más independientes, por lo que se da una tendencia a la motivación de logro (Ragúz, 1990), aspectos que son difíciles de satisfacer si se es VIH positiva.

A partir de la literatura consultada y por la experiencia de trabajo con mujeres seropositivas se sabe que la mayoría de éstas es contagiada por su pareja estable, en su hogar (Fasce, 1999; Fasce, 2001; Torres, 1998). En este estudio ninguna de las mujeres de la muestra ejercía como trabajadora sexual coincidiendo con investigaciones que las ubican como grupo de reducido índice de riesgo de contagio, lo cual contradice la creencia general que las ubica como muy expuestas (Estebañez et al., 1992).

El objetivo general de la presente investigación es identificar y describir los niveles de depresión en un grupo de adultos seropositivos asintomáticos y sintomáticos, así como encontrar las asociaciones significativas con los datos demográficos y estatus de salud de la muestra. Numerosas investigaciones señalan que la depresión se asocia con un mejor estatus de salud, pero dichas investigaciones han trabajado sólo 
con HSH (Antoni et al., 1991; Hays, Turner y Coates, 1992; Kelly y St. Lawrence, 1986; Kemmeny et al., 1994; Rabkin et al., 1991).

\section{Metodología}

La presente investigación se desarrolló como un estudio descriptivo, que buscó identificar los niveles de depresión en un grupo de adultos de uno y otro sexo, de nivel socioeconómico bajo y medio bajo, seropositivos en Lima (Hernández, Fernández y Baptista, 1996).

\section{Participantes}

El universo de estudio de la investigación estuvo conformado por la población de hombres y mujeres mayores de 18 años diagnosticados con VIH en Lima y que acuden a centros de salud, ONG o Grupos de Ayuda Mutua (GAM). Se utilizó un muestreo no probabilístico de tipo accidental. Los participantes fueron 55 personas adultas, hombres y mujeres, de 18 a 58 años de edad, VIH positivas, asintomáticas y sintomáticas, que se atienden en instituciones estatales de Lima. Se excluyeron a aquellos portadores que presentaban trastornos psíquicos graves, es decir, que se hallaban especificados en el eje I del DSM IV y aquellos que se ubicaban en fase SIDA (American Psychiatric Association,1994). En el Cuadro 1 aparecen las características más importantes de los participantes.

\section{Cuadro 1}

Descripción según estado civil y media de la edad

\begin{tabular}{|lccc|}
\hline Estado Civil & $f$ & $\%$ & $\begin{array}{c}\mathrm{X} \\
\text { Edad }\end{array}$ \\
\hline Soltero & 38 & 69 & 32 \\
Casado & 8 & 14 & 32 \\
Viudo & 1 & 2 & 44 \\
Conviviente & 7 & 13 & 30 \\
Separado & 1 & 2 & 46 \\
\hline
\end{tabular}

Nota. $N=55$. 
En el Cuadro 2 se observa que el $60 \%$ de las personas se halla entre los 24 y 35 años, así como el $66 \%$ de los hombres, mientras que las mujeres tienden a encontrarse en los extremos, encontrándose el $57 \%$ en el grupo de menores de 29 años, cumpliendo con esta característica sólo el $37 \%$ de los hombres; por otro lado, el 36\% de las mujeres son mayores de 41 años, cumpliendo con esta característica el $9 \%$ de los hombres.

\section{Cuadro 2}

Distribución los participantes por edad y sexo

\begin{tabular}{|cccc|}
\hline Edad & $\begin{array}{c}\text { Hombres } \\
\%\end{array}$ & $\begin{array}{c}\text { Mujeres } \\
\%\end{array}$ & $\begin{array}{c}\text { Total } \\
\%\end{array}$ \\
\hline$>23$ & 10 & 21 & 13 \\
$24-29$ & 27 & 36 & 29 \\
$30-35$ & 39 & 7 & 31 \\
$36-40$ & 15 & 0 & 11 \\
$41-46$ & 7 & 22 & 11 \\
$47-52$ & 2 & 7 & 4 \\
$53-58$ & 0 & 7 & 1 \\
\hline
\end{tabular}

Nota. $N=55$, mujeres $n=14$, hombres $n=41$

Referencialmente, la proporción hombre a mujer de la muestra obtenida se aproxima a la estadística oficial ( $3: 1)$ (Ministerio de Salud, 200 lb). Las mujeres constituyen el $26 \%$ de la muestra, confirmando observaciones respecto a la progresiva feminización de la epidemia por lo que se las considera grupo vulnerable (Kornblit, 1997, CONTRASIDA, 1998a).

De los datos de la descripción de la muestra, los más relevantes son aquellos que señalan las características de las personas que participan en un GAM (Cuadro 3). 
Depresión en personas que viven con VIH

\section{Cuadro 3}

Participación en un GAM por Orientación Sexual en Porcentajes

\begin{tabular}{|lcc|}
\hline Orientación sexual & \multicolumn{2}{c|}{ Participa en GAM } \\
\hline & Sí & No \\
Heterosexual & 48 & 52 \\
- mujeres & 50 & 50 \\
- hombres & 46 & 54 \\
Homosexual & 38 & 62 \\
- mujeres & 0 & 0 \\
- hombres & 38 & 62 \\
Bisexual & 29 & 71 \\
-mujeres & 0 & 0 \\
-hombres & 29 & 71 \\
\hline
\end{tabular}

Nota. $N=55$

\section{Instrumentos}

Los instrumentos usados fueron el Inventario de Depresión de Beck $(I D B)$ (Beck y Steer, 1993) y la Encuesta Demográfica (ED) (Fasce, 1999), ambos instrumentos fueron sometidos a criterio de jueces para obtener la validación de contenido y la adecuación lingüística. El $I D B$ fue sometido a análisis estadístico, obteniéndose un Alpha de Crombach de .86 para la prueba total, asimismo se obtuvieron cinco factores: (a) Déficit en la interacción social, con un Alpha de .72, (b) Cogniciones negativas, con un Alpha de .77, (c) Autoestima disminuida, con uno de .78 , (d) Incapacidad para disfrutar de los reforzadores, con uno de .56 y (e) Síntomas físicos asociados a la depresión, con un Alpha de .73 .

\section{Procedimiento}

Los instrumentos se aplicaron en cinco instituciones: una $O N G$, un GAM y tres hospitales. Esto se realizó mediante una entrevista oral, en forma individual con cada paciente. Cada día se evaluó a 
aproximadamente tres personas. En la ONG, los psicólogos de la institución se encargaron del trabajo. En los otros lugares, un grupo de tres psicólogos entrenados realizaron las entrevistas. En dos de los hospitales, al finalizar el trabajo se realizó un taller para los pacientes. Posteriormente, los datos de las entrevistas fueron sistematizados y analizados.

\section{Resultados}

Primero presentaremos los datos descriptivos relativos a los niveles de depresión recogidos con el IDB. Los resultados muestran que el $29 \%$ de los participantes presenta un nivel mínimo de depresión $(0-9)$, el $31 \%$ un nivel leve $(10-16)$, el $33 \%$ se halla con una depresión moderada (17-29) y sólo el 7\% con una depresión severa (superior o igual a 30). Estos resultados se grafican en la Figura 1.
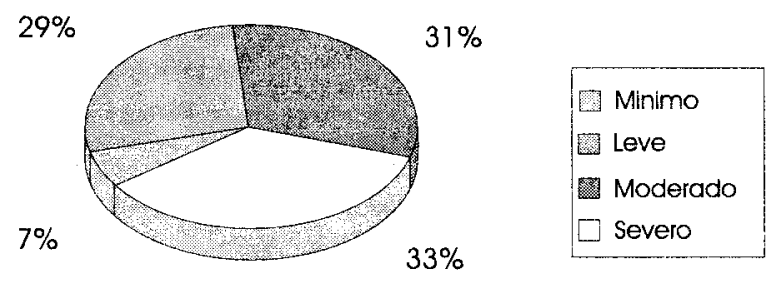

Figura 1. Descripción de los porcentajes de los índices del IDB.

La media en el IDB para aquellos que participan en un GAM fue leve $(M=12)$, mientras que para aquellos que no lo hacen fue moderada $(M=18)$, estos datos aparecen en el Figura 2 . 


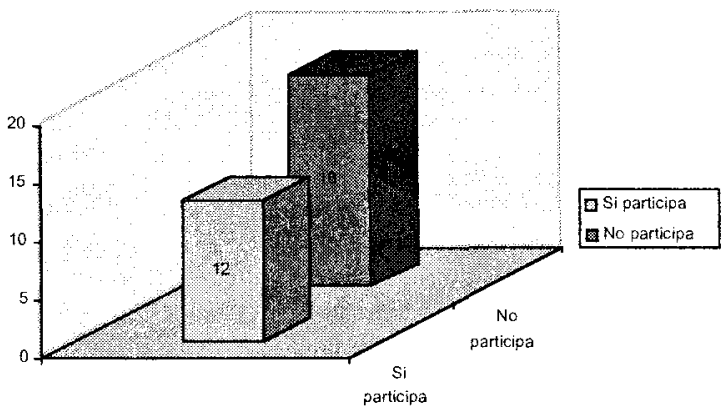

Figura 2. Índices del IDB según participación en el GAM.

Los niveles de depresión de las mujeres $(M=20)$ y los hombres que tienen sexo con otros hombres (HSH) $(M=17)$ fueron mayores que los niveles de depresión de los hombres heterosexuales $(M=8)$. Estos datos aparecen la Figura 3.
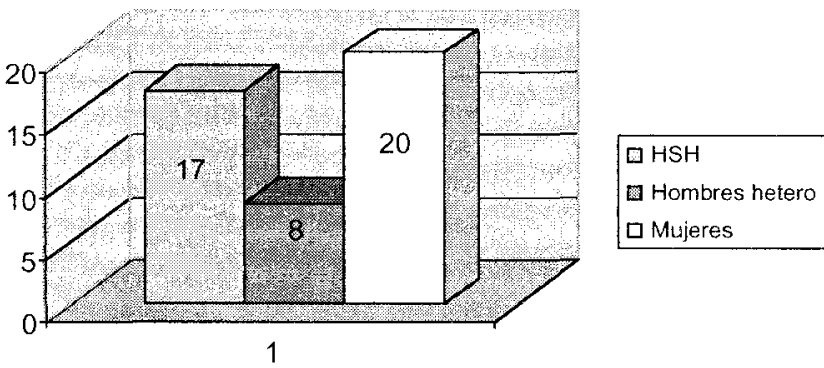

Figura 3. Índices del IDB por orientación sexual y sexo.

En esta parte presentaremos los resultados de las correlaciones entre niveles de depresión y variables demográficas. Para realizar esta parte llevamos a cabo un análisis factorial del IDB identificándose cinco factores:

- Factor 1: Déficit en la interacción social

- Factor 2: Cogniciones negativas 
- Factor 3: Déficit en la autoestima

- Factor 4: Incapacidad para disfrutar de los reforzadores

- Factor 5: Síntomas físicos.

El resultado total del IDB, así como los resultados de los cinco factores obtenidos fueron correlacionados con las variables demográficas de: participación en un GAM, sexo, orientación sexual y sexo, tiempo de diagnóstico, estatus de la enfermedad, si presenta o no síntomas y edad. La variable orientación sexual y sexo, se compuso de las siguientes variables: mujer heterosexual, hombre heterosexual y hombre homosexual.

Se observa que el participar en un GAM tuvo una gran asociación con el Factor 2 - Cogniciones Negativas ( $r=-.51$ ); es decir, si se pertenece a un GAM, se tendrán menos cogniciones depresivas. Asimismo, pertenecer a un GAM tuvo una asociación media $(r=-.34)$ con los niveles de depresión generales; en otras palabras, aquellos que participaron en un GAM tuvieron menores niveles de depresión que los que no participaron. El Factor 3 - Autoestima disminuida tuvo, también una asociación media con la participación en un grupo de autoayuda $(r=-.33)$.

Asimismo, el sexo de la persona tuvo una asociación media ( $r=-$ .35) con el Factor 5 -Síntomas físicos asociados a la depresión. Si se es mujer habrá más probabilidad de tener problemas físicos asociados a la depresión. Se encontró una asociación media aunque significativa $(r=-29)$ entre el sexo con los puntajes del inventario, así como con el Factor 1 -Déficit en las interacciones sociales $(r=-.28)$ de la depresión.

La presencia o no de síntomas asociados a la seropositividad tuvo una asociación media, aunque significativa con el IDB $(r=-.28)$, especialmente con el Factor 2 - Cogniciones negativas $(r=-.27)$, Factor 3 Autoestima disminuida $(r=-.26)$, y Factor 5 - Síntomas físicos asociados a la depresión $(r=-.27)$ de la depresión. 
La edad tuvo una correlación media e inversa con el Factor 4 Incapacidad para disfrutar de los reforzadores $(r=-.26)$, lo que significa que a más edad mayor capacidad para disfrutar de los reforzadores. Asimismo, la edad correlacionó positivamente con el Factor 5 - Síntomas físicos asociados a la depresión $(r=.25)$; es decir, a más edad mayores problemas físicos asociados a la depresión.

La orientación sexual y el tiempo del diagnóstico no tuvieron asociación alguna con el IDB Estos resultados se resumen en el Cuadro 4.

\section{Cuadro 4}

Asociaciones del IDB con las variables demográficas

\begin{tabular}{|ccccccc|}
\hline & $\begin{array}{c}\text { Participación en } \\
\text { un GAM }\end{array}$ & Sexo & $\begin{array}{c}\text { Orientación } \\
\text { sexual y sexo }\end{array}$ & $\begin{array}{c}\text { Tiempo de } \\
\text { diagnóstico }\end{array}$ & Sintomatología & Edad \\
\hline IDB & $-.34 *$ & $-.29 *$ & -.14 & -.02 & $-.28 *$ & .06 \\
Factor 1 & -.19 & $-.28 *$ & -.13 & .09 & -.04 & .16 \\
Factor 2 & $-.51 *$ & -.09 & -.20 & -.15 & $-.27 *$ & -.05 \\
Factor 3 & $-.33 *$ & -.22 & -.10 & .04 & $-.26 *$ & -.17 \\
Factor 4 & -.21 & -.09 & -.20 & .02 & -.01 & $-.26 *$ \\
Factor 5 & -.03 & $-.35 *$ & .02 & -.07 & $-.27 *$ & $.25 *$ \\
\hline
\end{tabular}

Nota. $N=55 . * p<.05$

\section{Discusión}

Se ensayó una aproximación a la comprensión de la depresión en personas seropositivas. El interés vino determinado por la conveniencia de apoyar a estas personas, debido a que la literatura señala que la presencia de niveles elevados de ansiedad y depresión ejercen efectos negativos sobre la salud y la respuesta ante los tratamientos (Antoni et al., 1991; Kemmeny et al., 1994; Rabkin et al., 1991). De aquí la importancia de conocer como se influencian las diferentes variables que afectan la salud, principalmente en los seropositivos, pues es la población con salud más frágil. Ello permitirá una mejor definición de los alcances y de la metodología del apoyo psicológico. 
La muestra está constituida por adultos de ambos sexos entre 18 y 58 años. Asimismo, el rango de edades se aproxima al $75 \%$ de personas VIH positivas entre 20 y 39 años propuesto para el Perú por la OPS (1999); cabe destacar que en este rango de edad, la mayoría de personas se encuentran trabajando. Ulin (1992) señala la importancia de considerar las consecuencias económicas de la epidemia a nivel social, pues no sólo el seropositivo pierde capacidad de trabajar, sino que genera gastos a su entorno cercano y a la sociedad.

Referencialmente, la proporción hombre-mujer de la muestra obtenida se aproxima a la estadística oficial (3:1). (Ministerio de Salud, 2001a). Las mujeres constituyen el $26 \%$ de la muestra.

Respecto a la orientación sexual, la muestra refleja el veloz incremento de seroprevalencia entre la población heterosexual en los últimos años (Kornblit, 1997), los contagios por vía heterosexual en el Perú entre 1995 y 1997 son del 53\%; mientras que los contagios por vía homosexual que son el $43 \%$. En la muestra los casos de heterosexuales alcanzan el $49 \%$, mientras que los homosexuales son el $51 \%$.

En cuanto a los objetivos de la investigación, se ha podido observar que la media de depresión de la muestra es de 15, es decir, un nivel leve de depresión, existiendo diferencias según el sexo y orientación sexual; los hombres que tienen sexo con otros hombres (HSH) y las mujeres presentan una depresión moderada, mientras los hombres heterosexuales presentan un nivel mínimo.

Es importante destacar que los índices de depresión varían según se pertenezca o no a un GAM, siendo los que tienen más tendencia a la depresión, aquellos que no participan de estos grupos. Esto nos lleva a considerar que pertenecer a un grupo de autoayuda es beneficioso para la población que vive con VIH, por lo que sería positivo enfatizar las investigaciones sobre por qué algunos grupos presentan 
menor probabilidad de participar espontáneamente, siendo ellos los de menor nivel de instrucción, menores de 24 años y mayores de 35 , y con más de 3 años de conocimiento de su diagnóstico. Estos últimos, especialmente, ya que tienen más probabilidades de presentar síntomas, lo que condiciona que la persona se deprima y experimente estrés, lo que, a su vez, tiene una influencia directa sobre el sistema inmune (Kennedy, Kielcot-Glaser y Glaser, 1990; Kielcot-Glaser y Glaser, 1995).

Se recomienda promocionar la participación en los GAM como una medida para amortiguar la depresión en las personas que viven con VIH. Sobre las asociaciones entre variables demográficas y el IDB, se destaca que el participar en un GAM reduce el índice de depresión en el grupo evaluado, disminuyendo principalmente las cogniciones negativas y aportando en la consecución de una mejor autoestima. Según Nezu, Nezu y Perry (Sanz y Vázquez, 1995) la depresión ocurre porque la persona atraviesa situaciones difíciles y/o negativas y no sabe como solucionarlas, debido a un déficit de estrategias de solución de problemas; así mismo, en la depresión ocurre que la persona magnifica lo negativo, con lo que el futuro se ve desesperanzador (Beck, 1990).

Parece ser que formar parte de un GAM brinda la oportunidad de conocer como otros han solucionado su problema y aprender estas estrategias, y la oportunidad de relacionarse con otras personas con el mismo problema, lo que ayuda a aprender que su situación no es tan terrible, ni catastrófica teniendo actitudes más positivas ante la vida. En los GAM se suele tratar continuamente el tema de la muerte, el cual genera ansiedad y depresión (Kelly y St. Lawrence, 1986). No obstante, enfrentarse continuamente al tema depresógeno los ayuda a desensibilizarse y manejar mejor su situación.

En cuanto al sexo, encontramos que el ser mujer se asocia con elevados índices de depresión, principalmente en el área de las inte- 
racciones sociales y los síntomas físicos; esto podría deberse a que la epidemia fue en un inicio una enfermedad de hombres por lo que se tiene mayor conocimiento de las enfermedades oportunistas en ellos lo que pude generar incertidumbre; asimismo, son las mujeres las que tienen mayor carga en el hogar por ser las que cuidan a los hijos y se encargan de la familia en general, lo que demanda energía, siendo ella escasa cuando se vive con VIH.

Esta información coincide con los datos epidemiológicos del Manual estadístico y diagnostico de los desordenes mentales DSM IV (American Psychiatric Association, 1994) cuarta edición, con respecto a la depresión, donde se refiere que la frecuencia en mujeres para depresión mayor es el doble que para hombres y el triple para distimia. Asimismo, los hombres suelen ser más asertivos, competitivos, con más capacidad aprendida para defender sus posiciones, ser líderes, correr riesgos, con más control sobre sus emociones y mayor confianza en sí mismos, lo que los protege de la depresión.

Con respecto a la edad, se observa que es un factor que se asocia positivamente en la muestra a los síntomas físicos de la depresión y negativamente a la incapacidad para disfrutar de los reforzadores. Esto coincide con lo que nos dice el DSM IV sobre la mayor prevalencia de síntomas tales como enlentecimiento psicomotor, hipersomnia y trastornos alimenticios en personas que se alejan de la adolescencia. Asimismo, tenemos que con el incremento de la edad las personas tienden a culparse y autocastigarse menos, siendo con los años menos propensos a privarse de satisfacciones que el medio les ofrece.

La aparición de los síntomas físicos asociados a la enfermedad se asocia en la muestra a índices mayores de depresión, especialmente en las áreas de las cogniciones negativas, autoestima disminuida y síntomas físicos de la depresión. nuestros hallazgos van en la línea de lo plateado por Kielcot-Glaser y Glaser (1995), quienes afirman 
que la aparición de síntomas condicionan altos niveles de ansiedad, cólera y depresión, vinculados con la aceleración del ingreso a la fase sintomática, debido a su influencia sobre el sistema inmune. Asimismo, Temoshok (1983) señala que las emociones y estados mentales, que se ven afectados con la aparición de síntomas, influyen en el estado de salud. Si la persona se ve ante eventos que no puede afrontar, como es el percatarse del deterioro de la propia salud, habrá un efecto negativo sobre el equilibrio social, emocional y por lo tanto, sobre su salud física (Hays et al.,1992; Hewitt y Flett, 1996 y Strelau, 1995).

Sería recomendable ampliar los estudios sobre las variables estudiadas en la población femenina, debido a que se comporta de manera diferente y el número de seropositivas al VIH se incrementa permanentemente, lo cual trae como consecuencia que existan casos de transmisión vertical (madre-niño) siendo el porcentaje $0.6 \%$ del total de gestantes en Lima. La mejor manera de prevenir la transmisión vertical sería reducir la infección en las mujeres en edad fértil, lo cual demanda de campañas de prevención focalizadas en ellas (Castro, 1998; CONTRASIDA, 1998b).

Se considera necesario ampliar las investigaciones, principalmente, con la población heterosexual pues la mayor parte de las investigaciones sobre el tema se realizan con una muestra de $\mathrm{HSH}$, y por lo que hemos podido observar, las necesidades no son iguales para los diferentes grupos de la muestra; las mujeres requieren de un trabajo diferente al de los hombres y las necesidades de ellos difieren según su orientación sexual, es por ello que se propone hacer programas de prevención por separado y que las intervenciones sea diferentes para cada grupo. Asimismo, sería idóneo se realicen investigaciones longitudinales e interdisciplinarias, donde se evalúe como el estatus de salud varía según varían otras áreas como el soporte social, el ánimo, los estilos de afrontamiento, el manejo de la cólera, así como, su relación con la personalidad. El conocer como interactúan las diferen- 
tes características de la población nos daría una visión más amplia de ella y las intervenciones podrían ser más cercanas a sus necesidades.

A partir del trabajo realizado podemos inferir algunas conclusiones generales interesantes:

- Las personas del estudio presentan, en su mayoría, niveles leves y moderados de depresión, a consecuencia de su situación de seropositividad y otros eventos personales.

- Los que participan en un GAM presentan niveles más bajos de depresión con relación a los que no acuden a ellos.

- Las mujeres y HSH presentan niveles de depresión mayores que los hombres heterosexuales.

- El sexo y la orientación sexual presentan asociaciones con el $I D B$, siendo las mujeres y los HSH los que muestran mayores niveles de depresión.

- El nivel de depresión se asocia a la existencia o no de síntomas asociados al VIH.

\section{Referencias}

American Psychiatric Association (1994). Diagnostic and Statiscal Manual of Mental Disorders (4ta. ed.). Washington DC: APA.

Antoni, M. H., Bagett, L. Ironson, G. La Perriere, A., August, S., Klimas, N., Schneiderman, N. y Fletcher, M. A., (1991). Cognitivebehavioral stress management interventions buffers distress responses and immunologic changes following notification of HIV1 seroposivity. Journal of Consulting and Clinical Psychology, 59(6), 906-915.

Beck, A. (1990). Tratamiento cognitivo de la Depresión. Bilbao: Brower.

Beck, A. y Steer, R. (1993). Beck Depression Inventory. San Antonio: The Psychological Corporation, Harcourt Brace \& Company. 
Castro O. P. (1998, mayo 10). Informe: Aumenta la esperanza de vida para hijos de portadoras del VIH. El Comercio, p. 18.

CONTRASIDA (1998a). Contrasida Informa. Boletín informativo del Programa de Enfermedades de Transmisión Sexual y SIDA, 7, 9.

CONTRASIDA (1998a). Contrasida Informa. Boletín informativo del Programa de Enfermedades de Transmisión Sexual y SIDA, 7,10 .

Estebañez, P., Rua-Figueroa, M., Aguilar, M., Fitch, K., Palacios, V., Pelez, L. y Nájera, R. (1992). Prevalencia de VIH y factores de riesgo entre prostitutas hispanas. Artículo presentado en la VII Conferencia Internacional sobre SIDA, Amsterdam.

Fasce, N. (1999). Depresión y soporte social en personas que viven con VIH. Tesis para optar el titulo de licenciado en Psicología con mención en Psicología Clínica, Pontificia Universidad Católica del Perú, Lima.

Fasce, N. (2001). Soporte social en personas que viven con VIH. Revista de Psicología de la Pontificia Universidad Católica del Perú, 19 (1), 66-86.

Hays, R. B., Turner, H. y Coates , T. (1992). Social Support, AIDSRelated symptoms, and Depression among gay men. Journal of Consulting and Clinical Psychology, 3, 463-469.

Hernández, R., Fernández, C. y Baptista, P. (1996). Metodología de la Investigación. México: McGraw-Hill.

Hewitt, P. y Flett, G. (1996). Personality traits and the coping process. En M. Zeidner y N. Endler, N. (Eds.), Handbook of coping: Theory, research, applications (pp. 410-433). Nueva York: Wiley.

Kelly, J. y St. Lawrence, J. S. (1986). Behavioral Intervention and AIDS. The Behavioral Therapist, 9 (6), 121-125.

Kemmeny, M. E., Weiner, H., Taylor, S. E., Schneider, S., Visscher, B. y Fahey, J. L. (1994). Repeated bereavement, depressed mood, and immune parameters in HIV seropositive and seronegative gay men. Health Psychology, 13, 14-24. 
Kennedy, J., Kielcolt-Glaser, J. y Glaser, R. (1990). Social Support, stress and the immune system. En B. Sarason, I. Sarason y G. Pierce (Eds.), Social Support: An interactional view (pp. 253266). Nueva York: Wiley.

Kielcot-Glaser, J. y Glaser, R. (1995). Psychoneuroimmunology and health consequences: Data and shared mechanism. Psychosomatic Medicine, 57, 269-274.

Kornblit, A. L. (1997, Junio). SIDA y ETS: Qué se ha hecho y qué se debe hacer. Artículo presentado en el IV Congreso Latinoamericano de Ciencias Sociales y Medicina, Coco y Mor, México.

Organización Mundial de la Salud (OMS) (1998). Resumen mundial sobre la pandemia VIH/SIDA. En linea: http://www.ctv.es/ USERS/fpardo/ vihdat. htm.

Organización Panamericana de la Salud (OPS) (2001). Boletín Epidemiologico, 21, 3.

Ministerio de Salud (2001a). Información estadística 1983-abril 2001 del programa de enfermedades de transmisión sexual y SIDA (PROCETSS). Lima: autor.

Ministerio de Salud (2001b). Información estadística 1983-abril 2001, del programa de enfermedades de transmisión sexual y SIDA (PROCETSS).

Rabkin, J. G., Williams, J. B. W., Remien, R. H., Goetz, R. R., Kretzner, R. y Gorman, J. M. (1991). Depression, lymphocyte subsets, and human immunodeficiency virus symptoms on two occasions in HIV-positive homosexual men. Archives of General Psychiatry, 48, 111-119.

Ragúz, M. (1990). Maternidad / Maternalidad y Trabajo: "Efectos" del Rol Dual sobre las Madres que Trabajan. Revista de Psicología de la Pontificia Universidad Católica del Perú, 8 (2), 181-197.

Ragúz, M. (1995). Aportes de Género a la Salud Sexual y Reproductiva. Revista de Psicología de la Pontificia Universidad Católica del Perú, 8 (1), 17-33.

Sanz, J. y Vázquez, C. (1995). Trastornos del estado de ánimo: Teorías psicológicas. En A. Belloch, B. Sandin y F. Ramos (Eds.), 
Manual de Psicopatología (Vol. 2). Madrid: McGraw-HillInteramericana de España.

Strelau, J. (1995). Temperament and Stress: temperament as a moderator of stressors, emotional states, coping and cost. En C. D. Spielberg, I. Sarason, J. Brebner, E. Grunglass, P. Laungani y A. O'Roark (Eds.), Stress y emotion: Anxiety, anger and curiosity (Vol. 15). Washington: Taylor y Francés.

Temoshok, A. (1983). Emotion, adaptation, and disease: A multidimensional theory. En L. Temosohk, C. Van Dyck, y L. S. Zegans (Eds.), Emotions in health and illness: Theoretical and Research Foundations (pp. 235-257). Nueva York: Grune y Stratton.

Torres, G. (1998). Oye nuestras voces. ICW Noticias, 7, 2.

Ulin, U. (1992). African woman and AIDS: Negotiating behavior change. Social science and medicine, 34, 63-73. 